\title{
Diseño de una fuente conmutada y galvanómetro digital para calibración de respuesta angular de sensores de radiación solar
}

\section{Switching power supply and digital galvanometer design for calibrating an angular response of solar radiation sensors}

\author{
Nelson Díaz Spencer ${ }^{1} \quad$ Leonardo Fernández Castillo ${ }^{2} \quad$ Juan C. Antuña Sánchez ${ }^{3} \quad$ Jorge Rosas Santana ${ }^{4}$ \\ Albert Rodríguez Vega $\quad$ Frank García Parrado $\quad$ Iralmy Y. Platero Morejón ${ }^{7}$ \\ Instituto de Meteorología, Cuba
}

\section{RESUMEN}

Con el objetivo de determinar el factor de corrección por altura del sol, en los piranómetros - albedómetros empleados en las estaciones actinométricas de Cuba para las mediciones de irradiancia solar, se ha instalado en el Grupo de Óptica Atmosférica de Camagüey un Sistema de Calibración de Respuesta Angular para sensores de radiación solar. Las mediciones realizadas en estas estaciones tributan proveen datos al Servicio de Diagnóstico de Radiación Solar para Cuba. La instalación de este sistema, único de en su tipo en el país, ha sido posible a través de un proyecto de colaboración con el Grupo de Óptica Atmosférica de la Universidad de Valladolid. Para el empleo de este sistema ha sido necesario diseñar y construir tanto, la fuente conmutada de alimentación para la lámpara halógena empleada para las calibraciones, y un galvanómetro digital para registrar los datos generados. El objetivo del presente trabajo es por tanto el diseño, construcción y puesta a punto de funcionamiento de ambos dispositivos. En el caso de la fuente conmutada se estudiaron fuentes con similares prestaciones existentes en el mercado, a partir de la cuales se procedió al diseño electrónico y simulación de los circuitos correspondientes. Finalmente, tanto la fuente conmutada como el galvanómetro digital fueron construidos $y$ actualmente se encuentran en fase de validación. Este último instrumento será replicado para sustituir a sus similares analógicos en el Servicio de Diagnóstico de Radiación Solar para Cuba.

Palabras clave: Calibración, respuesta angular, fuente conmutada, galvanómetro.

\section{ABSTRACT}

With the objective to determine the sun altitude correction factor, for the pyranometers - albedometers used in the Cuban actinometric stations for solar irradiance measurements, an Angular Response Calibration System for solar radiation sensors has been installed in the Atmospheric Optics Group of Camagüey. The performed measurements in these stations provide data to the Solar Radiation Diagnostic Service for Cuba. The installation of this system, unique of its kind in the country, has been possible through a collaboration project with the Atmospheric Optics Group of Valladolid University (GOA-UVA). For the usage of this system, it has been necessary to design and construct both, a switching power supply for the halogen lamp used in calibrations, as well as a digital galvanometer to collect the generated data. The objective of this research is the design, construction and operating tune of both devices. In the case of the switching power supply, power sources with similar features in the market were studied, from which we proceeded to the electronic design and simulation of the corresponding circuits. Finally, both the switching power supply and the digital galvanometer were built and nowadays are being validated. The last one device will be replicated in order to replace its analogic homologues in the Solar Radiation Diagnostic Service for Cuba.

Keywords: Calibration, angular response, switching power supply, galvanometer.

Historial del artículo:

Recibido: 14 de agosto de 2015. Aprobado: 19 de noviembre de 2015. Disponible en línea: 30 de diciembre de 2015

1 Ing. Electricista, Ingeniero del Grupo de Óptica Atmosférica de Camagüey, INSMET, Cuba. nelson@goac.cu

2 Ing. Electricista, Ingeniero del Centro Nacional de Radares, Centro Meteorológico Provincial de Camagüey, INSMET, Cuba.

3 Ing. en Ciencias Informáticas, Ingeniero del Grupo de Óptica Atmosférica de Camagüey, INSMET, Cuba.

4 Lic. en Meteorología, Especialista en Meteorología del Grupo de Óptica Atmosférica de Camagüey, INSMET, Cuba.

5 Mag. en Ciencias Meteorológicas, Especialista en Meteorología del Grupo de Óptica Atmosférica de Camagüey, INSMET, Cuba.

6 Lic. en Meteorología, Especialista en Meteorología del Grupo de Óptica Atmosférica de Camagüey, INSMET, Cuba.

7 Lic. en Meteorología, Especialista en Meteorología del Grupo de Óptica Atmosférica de Camagüey, INSMET, Cuba. 


\section{INTRODUCCIÓN}

Por más de 20 años el Grupo de Óptica Atmosférica de Camagüey (GOAC), Cuba, ha realizado investigaciones vinculadas con la óptica atmosférica y la radiación solar., entre otras (1). Para las mediciones de la radiación solar en superficie se han empleado las estaciones actinométricas manuales, de fabricación soviética, del tipo Yanishevski (2). Los datos generados a partir de estas mediciones han sido digitalizados a través de procesos de rescate de información (3) con el objetivo de ser empleados en las investigaciones que lleva a cabo el $\operatorname{GOAC}(4,5,6)$. Estas estaciones actinométricas están conformadas por los siguientes sensores:

1. Actinómetro: medición de la irradiancia directa.

2. Albedómetro (piranómetro): medición de la irradiancia global, difusa y reflejada por la superficie terrestre.
Radiación Solar para Cuba (SDRS) (9). En la estación de Casablanca aún no ha sido instalado el software correspondiente por lo que no tributa sus datos al SDRS.

De todos los sensores que conforman estas estaciones actinométricas el más crítico es el piranómetro, que por sus características constructivas, es el más frágil. Una gran cantidad de los existentes están fuera de servicio, ya sea por el deterioro de la superficie ajedrezada cuadriculada o por la rotura de la cúpula de cristal que protege al sensor. Su recuperación es por tanto de máxima prioridad y requiere la calibración angular del mismo debido a la heterogeneidad de la cúpula de cristal. Hasta el presente, la única calibración que se realiza a estos sensores en el país es por el método de comparación con un patrón homologado y bajo condiciones de cielo despejado. Estas calibraciones no incluyen la determinación del factor de calibración angular.

En Estados Unidos de América el Sistema Medio

Tabla $N^{\circ}$ 1: Estaciones actinométricas instaladas en Cuba, de éstas, sólo las cuatro primeras (verde) forman parte del SDRS para Cuba.

\begin{tabular}{cllcrr}
\hline Código & \multicolumn{1}{c}{ Nombre } & \multicolumn{1}{c}{ Provincia } & Latitud & Longitud & Altitud $(\mathrm{m})$ \\
\hline 321 & Santa Fe & Isla de la Juventud & 21,733 & 82,767 & 31,72 \\
330 & Jovellanos & Matanzas & 22,420 & 81,167 & 25,23 \\
342 & Topes de Collantes & Sancti Spíritus & 21,919 & 80,016 & 766,35 \\
355 & Camagüey & Camagüey & 21,421 & 77,871 & 118,00 \\
325 & Casablanca & La Habana & 23,143 & 82,342 & 50,80 \\
\hline
\end{tabular}

3. Balanzómetro: medición del balance radiativo terrestre.

Todos estos sensores están conectados a uno o más instrumentos analógicos conocidos como galvanómetros, encargados de registrar los pequeños voltajes generados por los termopares de los sensores antes mencionados (7). Tanto los sensores como los instrumentos de medición que forman parte de estas estaciones, cuentan con más de 40 años de explotación (8), por lo que el sostenimiento de estas mediciones es una labor con grandes inconvenientes. Debido al alto costo, tanto de los sensores como de los registradores de las versiones más modernas de estos instrumentos, no ha sido posible la renovación de estos en las estaciones actinométricas del país.

En Cuba se cuenta con 5 estaciones actinométricas desplegadas en diferentes regiones del país para la medición de la radiación solar en superficie (tabla 1). De estas estaciones, solamente las cuatro primeras forman parte del Servicio de Diagnóstico de
Ambiental Yankee (YES, por sus siglas en ingles), ofrece servicios de calibración óptica para instrumentos de radiación solar, donde caracterizan la respuesta espectral, absoluta y angular de estos instrumentos (10), pero estos servicios, al tener un alto coste, no son accesibles para el país. El Grupo de Óptica Atmosférica de la Universidad de Valladolid, España (GOA-UVA), ha colaborado con el Observatorio Atmosférico de Izaña para la creación de un laboratorio de calibración óptica. Este laboratorio cuenta con 6 sistemas de calibración de instrumentos de medidas de radiación, dos de estos sistemas son empleados para medir la irradiancia absoluta tanto de forma vertical como horizontal (11).

A través del convenio de colaboración existente entre el GOAC y el GOA-UVA, se ha construido en las instalaciones de este último, un sistema de calibración horizontal de similares características al emplazado en Izaña. Este sistema ha sido instalado en el Laboratorio de Calibración de Respuesta Angular del GOAC y se empleará para la calibración angular de los 
piranómetros - albedómetros que forman parte de las estaciones actinométricas de Cuba. El sistema, hasta donde se conoce, sería el primero y el único de su tipo en Centroamérica y el Caribe.

El elemento activo de este sistema de calibración está constituido por una lámpara halógena de alta estabilidad y precisión, la cual requiere de una fuente de alimentación que cumpla con las especificaciones de alta estabilidad y muy alta regulación de tensión $(\% \Delta \mathrm{V})$. Las fuentes con estas características y para este fin tienen un alto costo en el mercado, oscilando entre los $\$ 4,000$ y $\$ 12,000$ USD. Tal es el caso de la fuente Oriel Digital Radiometric Power Supply modelo 69931. Pese a la falta de recursos existentes, el GOAC se propuso construir una con similares características y a un costo menor.

Para realizar las mediciones de los valores de intensidad relativa de la radiación, que registran los sensores sometidos al proceso de calibración, se emplearía un galvanómetro analógico, los mismos que son empleados en las estaciones actinométricas. Luego de un análisis de las características del mismo y de las condiciones en que se realizarán las calibraciones, se concluyó que debería emplearse un instrumento digital. Sin embargo, GOAC no cuenta con dicho instrumento, por lo que para dar solución a esta problemática se construyó un prototipo digital. Este prototipo garantizará una correcta toma de datos durante el proceso de la calibración, además, este mismo galvanómetro podrá ser replicado para que sea emplazado en las estaciones actinométricas y mejorar por tanto la calidad de las lecturas en las observaciones de radiación solar.

\section{MATERIAL Y MÉTODOS}

El primer paso para la construcción de la fuente fue encontrar el transformador adecuado, capaz de entregar la potencia exigida por la carga. Se colocaron dos autotransformadores con las mismas características en paralelo, el primario se alimentará con el voltaje de línea, entregado por el secundario de los transformadores 26 volt de corriente alterna (AC). Esto será en la etapa de reducción de AC. Para convertir $A C$ en corriente continua (DC) pulsante se necesitaron 4 diodos de silicio colocados en forma de puente para realizar una rectificación de onda completa. Dado que la DC no es pura, es decir, contiene armónicos; ésta es filtrada con un capacitor de altos valores de capacitancia, $4000 \mu \mathrm{f} / 50 \mathrm{~V}$, con el fin de eliminar los armónicos de baja frecuencia que se encuentran por encima de la frecuencia de la línea.

\section{Fuente conmutada variable para alimentar la lámpara del sistema de calibración}

Tomando como referencia la Oriel Digital Radiometric Power Supply modelo 69931 y sus características, se diseñó y construyó una fuente conmutada con similares características. El diseño se realizó sobre la base de los elementos y dispositivos electrónicos disponibles, la mayor parte de ellos, recuperados de las partes útiles del LIDAR que operaba el GOAC y de partes descontinuadas de los radares meteorológicos digitalizados por el Centro Nacional de Radares de Cuba. En la figura 1 se representa el diagrama en bloques de la fuente regulada conmutada que tiene las siguientes características principales:

- Tensión de alimentación o línea (Vin): 198 - 240 VACrms.

- Tensión de salida (Vout): 24 VDC.

- Potencia de salida (Pout): 250 W.

- Regulación de tensión (\% $\Delta \mathrm{V}): 0,2 \%$.

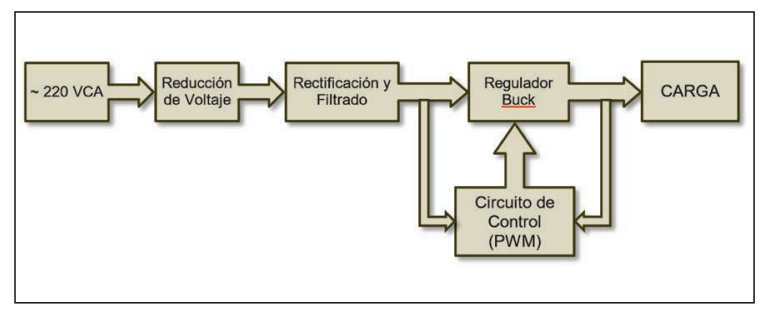

Figura $N^{\circ}$ 1: Diagrama en bloques de la Fuente Regulada Conmutada.

La regulación de tensión (\% $\Delta \mathrm{V}$ ) es un requisito fundamental con el cual debe cumplir la fuente para garantizar la estabilidad del voltaje en la lámpara durante el proceso de calibración. La ecuación para determinar este parámetro está dada por la siguiente expresión:

$$
\% \Delta V=\frac{(V s c-V c)}{V c} \cdot 100 \% \leq 10 \%
$$

Donde:

VSC: Tensión a la salida sin carga

VC: Tensión a la salida con carga

La lámpara, según el fabricante, debe ser alimentada con 24 VDC y demandará hasta 10A de corriente, parámetros con los que debe cumplir la fuente. Luego de realizar el diseño preliminar se buscaron los elementos y dispositivos para construir cada uno de los bloques de la fuente. 


\section{RESULTADOS}

La figura 2 muestra el circuito eléctrico del regulador Buck (reductor de tensión, Vout $<$ Vin), esta es la etapa que define a la fuente conmutada, este bloque está compuesto por el transistor de potencia, en este caso son 5 transistores en paralelo de $3 \mathrm{~A}$ cada uno, un diodo de marcha libre Dm y el filtro L-C, compuesto por un inductor $(L)$ en serie y un capacitor en derivación (C). El filtro $\mathrm{L}-\mathrm{C}$ es el responsable de mantener alimentada la carga cuando los transistores no estén en conducción. Como se mencionó anteriormente respecto al origen de los componentes de la fuente, los transistores

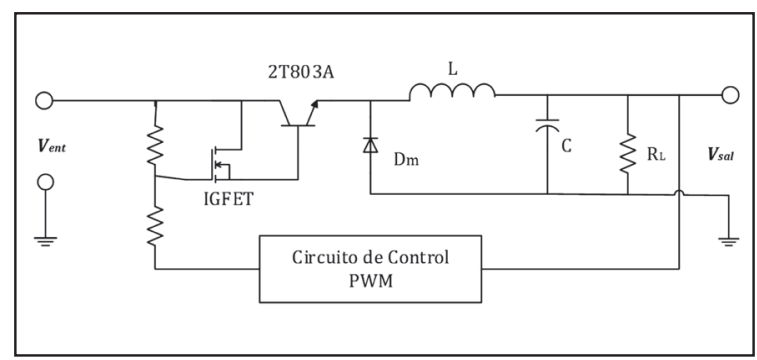

Figura $N^{\circ}$ 2: Regulador Conmutado Reductor controlado (Buck).

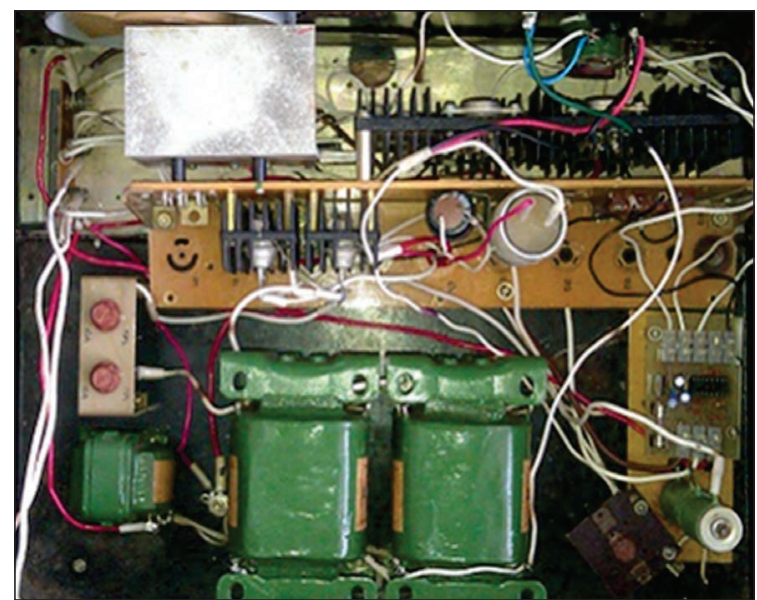

Figura $\mathrm{N}^{\circ}$ 3: Fuente Conmutada Variable.

de potencia, modelo $2 \mathrm{~T} 803 \mathrm{~A}$, formaban parte de un bloque de los radares soviéticos que han sido modernizados. Esas mismas condiciones las cumplen el filtro L-C.

El circuito de control está conformado por el circuito integrado $(\mathrm{Cl})$ PWM SG3524, este $\mathrm{Cl}$ reúne las características necesarias para la construcción de una fuente tipo Push - Pull. Este $\mathrm{Cl}$ controla un transistor del tipo IGFET tipo N, el que a su vez, controla los transistores de potencia. Finalmente, para comprobar la fiabilidad, estabilidad y regulación de la tensión de la fuente, se utilizó un banco de resistencias como carga, con un valor similar a la carga correspondiente a la lámpara a utilizar en el proceso de calibración. En la figura 3 se puede apreciar la fuente concluida con su estructura interna y componentes conectados.

\section{Galvanómetro Digital}

Para la construcción y diseño del galvanómetro digital, se tuvo en cuenta primeramente la amplificación de la señal proveniente del sensor, dada que ésta es muy pequeña, en el orden de 3 a 6 milivoltios $(\mathrm{mV})$, alcanzando valores de hasta $10 \mathrm{mV}$. Estas son conocidas como señales de bajo modo común, por lo que se empleó un amplificador de instrumentación (AI) con un alto factor de rechazo de modo común (CMRR

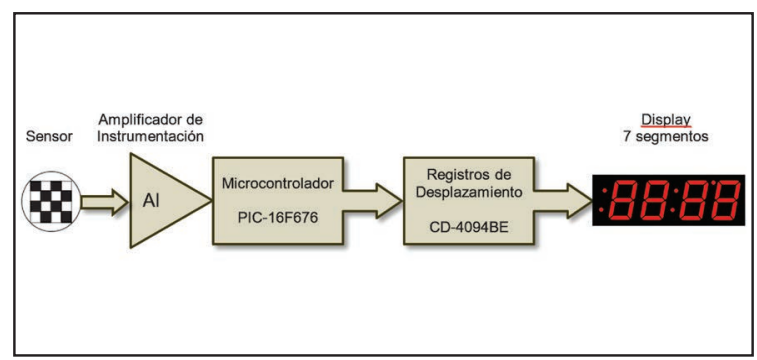

Figura $\mathrm{N}^{\circ}$ 4: Diagrama en bloques del Galvanómetro Digital.

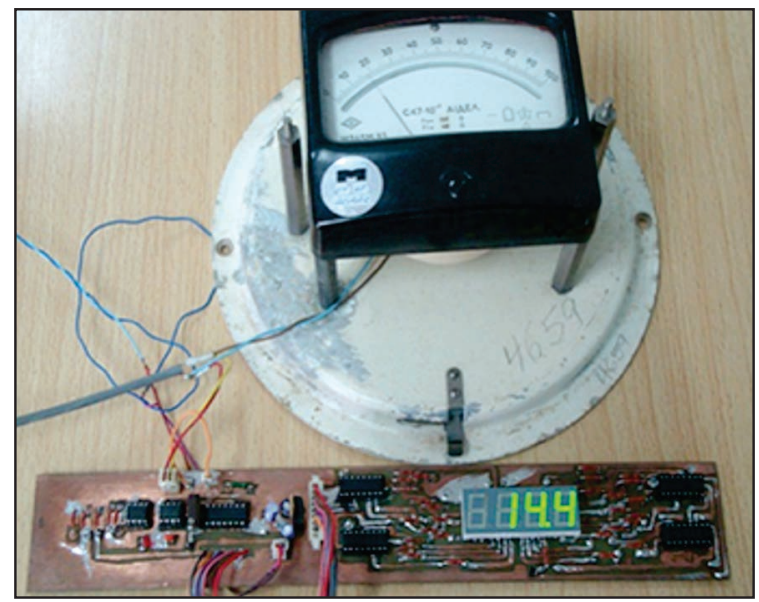

Figura $N^{\circ}$ 5: Prototipo del galvanómetro digital frente al galvanómetro analógico durante una medición de prueba.

- Common Mode Rejection Ratio). El Al utilizado fue AD623AN el cual cuenta con un muy buen CMRR, además, tiene la facilidad de alimentarlo con fuente simple, (una batería), disminuyendo así su costo.

La señal analógica amplificada va al microcontrolador PIC16F676 que cuenta internamente con un módulo convertidor Analógico - Digital (A/D) de 10 bit de resolución, 8 canales de entradas programables y una entrada de voltaje de referencia. La visualización de las mediciones se realiza a través de un display 7 segmentos de ánodo común de 4 dígitos que estará controlado 
por 4 registros de desplazamiento CD4094BE. La transmisión de la información proveniente del PIC hacia los registros de desplazamientos se realiza en serie.

El galvanómetro cuenta con un rango de medición de -99 mV a $100 \mathrm{mV}$, siendo estos los valores mínimos y máximos respectivamente. Para la puesta a punto del galvanómetro se realizaron varias pruebas por separado, es decir, separando los diferentes bloques que lo componen. Primero la amplificación de la señal, luego la programación del PIC donde se garantiza la conversión $A / D$ y la transmisión del dato para su visualización, se chequeó la correcta comunicación desde el PIC hacia los registros de desplazamientos y estos con el display 7 segmentos.

Para cerrar el ciclo se hacen varias series de mediciones con el prototipo digital y su par analógico conectados ambos al mismo sensor. Esto se realiza con el fin de ajustar el prototipo digital al patrón analógico que es utilizado actualmente para calibrar las estaciones actinométricas. La figura 4 muestra el prototipo del galvanómetro digital frente al galvanómetro analógico.

\section{Fuente Conmutada Variable}

Para tres valores distintos de cargas, la fuente arrojó tres valores distintos de tensiones medidos a la salida de la misma. La siguiente tabla muestra estas mediciones para cada una de las cargas.

Los resultados obtenidos (mostrados en la tabla anterior), demostraron que la fuente aun no cumplía con los requisitos planteados. Estos resultados se deben a que en la fuente existe una caída de tensión, de aproximadamente $4 \mathrm{~V}$, entre la salida del secundario de los autotransformadores y el capacitor de filtrado de baja frecuencia, es decir, en el puente de diodos rectificador. Esto provoca que a menor valor de tensión la intensidad de corriente aumente y le exija más a los autotransformadores provocando como consecuencia, el calentamiento en los mismos. La solución más simple fue elevar el valor de la tensión de salida del secundario cambiando la posición de las derivaciones. La tensión de salida, que en principio era de 26 VAC, se elevó a 30 VAC, de esta forma se garantizó que a la salida de la fuente, independientemente del valor de la carga, la tensión de salida sea de 24 VDC.

\section{DISCUSIÓN}

Entre los principales requisitos con los que debía cumplir el galvanómetro digital se encontraban la estabilidad del valor medido en el display, así como, un buen ajuste con su par analógico. El próximo paso consistió en llevar el prototipo de galvanómetro digital junto al galvanómetro analógico que se encontraba operativo en la estación actinométrica de Camagüey.
Las mediciones realizadas en paralelo permitieron comprobar que el funcionamiento del galvanómetro digital es correcto. La estructura final donde quedará montado el prototipo de galvanómetro digital no se ha construido aún, pero con el prototipo actual se realizaron pruebas que arrojaron resultados satisfactorios.

En conclusión, a pesar de los pocos recursos con los que se contaba se pudieron construir la fuente de alimentación de la lámpara para la calibración angular de instrumentos de radiación solar y el galvanómetro digital para la medición de los valores de intensidad relativa de la radiación que registran los sensores sometidos a calibración. Considerando que en ambos casos quedan mejoras por realizar.

El prototipo desarrollado permitirá mejorar y replicar este instrumento para ser empleado en las estaciones actinométricas de Cuba. La construcción de este instrumento significará un considerable ahorro de divisas al país por concepto de importación de equipos con similares características. Las lecturas que se realizan durante las mediciones de irradiancia solar experimentarán un salto de calidad considerable, al

Tabla $N^{\circ}$ 2: Valores medidos a la salida de la fuente con carga.

\begin{tabular}{ccccc}
\hline $\begin{array}{c}\text { No. de } \\
\text { pruebas }\end{array}$ & $\begin{array}{c}\text { Voltaje } \\
\text { salida }(\mathrm{V})\end{array}$ & $\begin{array}{c}\text { Corriente } \\
\text { carga }(\mathrm{A})\end{array}$ & $\begin{array}{c}\text { Carga } \\
(\Omega)\end{array}$ & $\% \Delta \mathrm{V}(\%)$ \\
\hline 1 & 17,15 & 9,93 & 2,20 & 43,40 \\
2 & 21,40 & 6,68 & 3,30 & 15,00 \\
3 & 20,40 & 7,87 & 2,70 & 20,50 \\
\hline
\end{tabular}

eliminar errores comunes en los procedimientos de medición como el error de paralaje y de redondeo, entre otros.

\section{Agradecimientos}

Al Grupo de Óptica Atmosférica de la Universidad de Valladolid por su apoyo en la construcción del sistema de calibración. Al Centro Nacional de Radares de Cuba por facilitar parte de los elementos y componentes empleados en la construcción de la fuente conmutada. Al Dr. Luis Vidal Ponce Cabrera, profesor de la Facultad de Física de la Universidad de la Habana, por donar los componentes utilizados en la construcción del galvanómetro digital.

\section{REFERENCIAS BIBLIOGRÁFICAS}

1. Antuña JC, Estevan R, Barja B. Demonstrating the Potential for First-Class Research in Underdeveloped Countries: Research on Stratospheric Aerosols and 
Cirrus Clouds Optical Properties, and Radiative Effects in Cuba (1988-2010). Bull. Amer. Meteor. Soc. 2012; 93(7): 1017-1027.

2. Estevan R, Antuña JC, Barja B, Hernández CE, Hernández TA, Acea R. Servicio de diagnóstico de radiación solar para Cuba. Camagüey: Grupo de Óptica Atmosférica de Camagüey / Instituto de Meteorología; 2011.

3. Antuña JC, Fonte A, Estevan R, Barja B, Acea $R$, Antuña Jr JC. Solar radiation data rescue at Camagüey, Cuba. Bull. Amer. Meteor. Soc. 2008; 89(10): 1507-1511

4. Fonte A, Antuña JC. Caracterización del espesor óptico de banda ancha de los aerosoles troposféricos en Camagüey, Cuba. Revista Cubana de Meteorología. 2011 ; 17(1): 15-26.

5. García F, Estevan R, Antuña-Marrero JC, Rosas J, Platero IY, et al. Determinación de la Línea Base del Espesor Óptico de Aerosoles de Banda Ancha y comparación con datos de fotómetro solar. Opt. Pura Apl. 2015; 48(4): 249-258.

6. Iralmy IY, Estevan R, García F. Climatología del albedo de superficie en la Estación Actinométrica de Camagüey. Opt. Pura Apl. 2015; 48(4).

7. Grupo de Óptica Atmosférica de Camagüey.
Manual de procedimiento para Observadores Actinométricos. Grupo de Óptica Atmosférica de Camagüey, Instituto de Meteorología de Cuba; 2010.

8. Antuña JC, Hernández CE, Estevan R, Barja B, Fonte A, et al. Camagüey's solar rescued dataset: Preliminary applications. Opt. Pura Apl. 2011; 44(1): 41-46.

9. Servicio de Diagnóstico de Radiación Solar [Internet]. Camagüey: Grupo de Óptica Atmosférica de Camagüey c2010 [actualizado 1 de abril de 2015; citado el 29 de julio de 2015]. Disponible en: http://www.goac.cu/actino/.

10. Yankee Environmental Systems [Internet]. Massachusetts: Yankee Environmental Systems, Inc.; c 1998-2006 [actualizado el 11 de septiembre de 2006; citado el 29 de julio de 2015] Optical Calibration Services [1 pantalla]. Disponible en: http://www.yesinc.com/support/optcal/optcal. html.

11. Guirado C, Ramos R, Frutos Á de, Berión A, Redonda A, et al. Optical calibration facility at the Izaña Atmospheric Research Center. Opt. Pura Apl. 2012; 45(1): 57-62. 